\title{
Expression du gène du TGF-ß dans un modèle expérimental de fibrose radioinduite
}

\author{
M. MARTIN, F. CRECHET, P. PINTON, J.-L. LEFAIX, F. DABURON \\ Laboratoire de radiobiologie appliquée, Commissariat à l'énergie atomique, \\ DSV/DPTE, 91191 Gif-sur-Yvette Cedex
}

Dans un modèle expérimental chez le porc, une irradiation gamma de la peau produit une fibrose cicatricielle. Nous avons testé dans ce modèle l'hypothèse selon laquelle une production anormale du facteur de croissance TGF- $\beta$ dans les tissus irradiés provoquerait une activation chronique des cellules responsables de la fibrose.

Pendant la phase d'inflammation précédant la formation de la fibrose, l'expression du gène codant le TGF-B1 est fortement augmentée dans la peau irradiée et varie suivant la dose délivrée aux tissus (140 à 14 Gy, dose unique). Six mois après irradiation, alors que le tissu fibreux cicatriciel est formé, une expression importante du TGF-B1 est retrouvée dans la peau cicatrisée et dans la fibrose sous-cutanée et musculaire. D'autre part, des cultures primaires de fibroblastes isolés de tissu fibreux expriment le facteur à un taux très supérieur à celui de cultures de fibroblastes témoins. Cette surexpression est maintenue jusqu'à 2 ans après irradiation.

Ces résultats impliquent le TGF- $B$ dans les processus de formation de la fibrose radique. Pendant la phase précoce d'inflammation, le facteur est probablement sécrété par les plaquettes sanguines et par les macrophages. Pendant la phase d'extension chronique de la fibrose, le TGF-b serait synthétisé par les fibroblastes eux-mêmes, dans un mécanisme de stimulation autocrine. 